\title{
Simulation Aided Process for Developing Powertrains
}

\author{
SAE Convention, Sao Paulo Brasil, October 2000 \\ Thomas Lang, Christian Schyr, AVL List GmbH Graz
}

\begin{abstract}
For the development of complex control algorithms and strategies the engine and powertrain test bed offers a number of advantages over the development in the prototype vehicle. The paper discusses how state-of-the-art simulation techniques can contribute to a continuous development process, which is based upon off-line simulation using hardware in the loop, the utilization of modern test bed technology up to vehicle adjustment. The integration of hardware-in-the-loop testing together with vehicle and transmission simulation on the testbed allows to speed up the optimization of fuel consumption, emissions and driveability in an early stage in the development process. The available software tools are presented and application examples are given.
\end{abstract}

\section{Introduction}

Each introduction of new methodologies in the powertrain development processes has to be aimed at reducing cost and development times. The process described in this paper is mainly based upon the following considerations:

- The development with and in the vehicle is expensive and very time consuming and should be reduced as far as possible.

- Development work on the test bed should be shifted to simulation as far as possible, i.e. vehicle tests $->$ work on the test bed $->$ offline simulation

- The development of all the systems and their combinations should be performed in parallel as soon as possible

- Failures should be avoided and corrected as soon as possible

In figure 1 an idealized development process is shown, which has been divided into four stages. After the computer simulation of the total vehicle, the share of the components physically present is continuously increasing up to the actual vehicle from the left to the right while the share of simulation is decreasing [1].

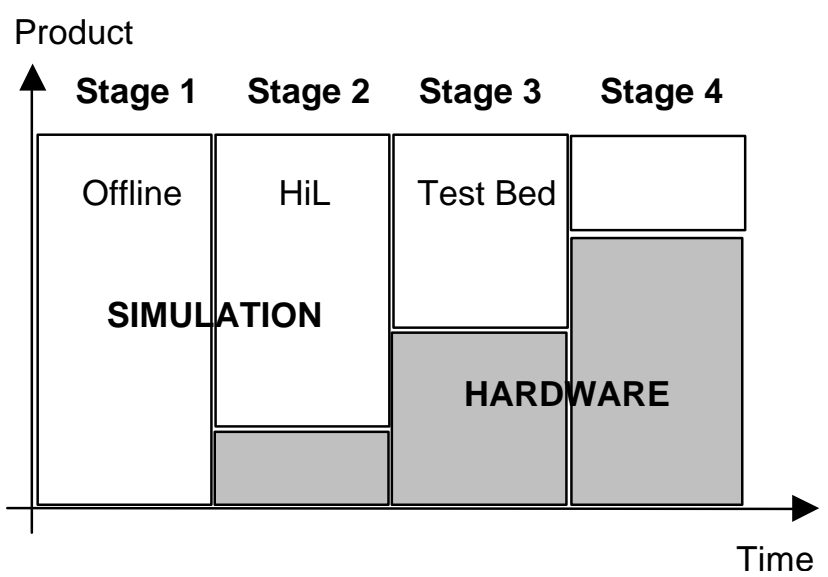

Figure 1: Four-Stage Development Process

\section{Offline Modelling and Simulation}

The offline simulation of the planned vehicle or the vehicle to be improved is at the beginning of the development process. After a preselection of powertrain components, this stage serves to analyze the powertrain. The following development work can be performed:

- Computing driving performance (maximum speed, acceleration, elasticity)

- Predicting fuel consumption and emissions

- Optimizing parameters (dimensioning power, transmission ratios, gear shifting strategies)

- Comparing powertrain concepts (conventional, continously variable transmission, hybrid, fuell cell) 
- Determining sets of loads for computing or measuring durability

- Minimizing the number of variants (at a large product variety)

The final products of this stage will be clear specifications for the powertrain components to be developed or purchased.

In modern offline simulation software packages a graphical user interface is provided to edit the vehicle model and is shown in figure 2 . The user can select between a wide range of model elements and also incorporate his specific coding. The results of the offline simulation are stored in a database and are available to be used in the next stages of the development process [2].

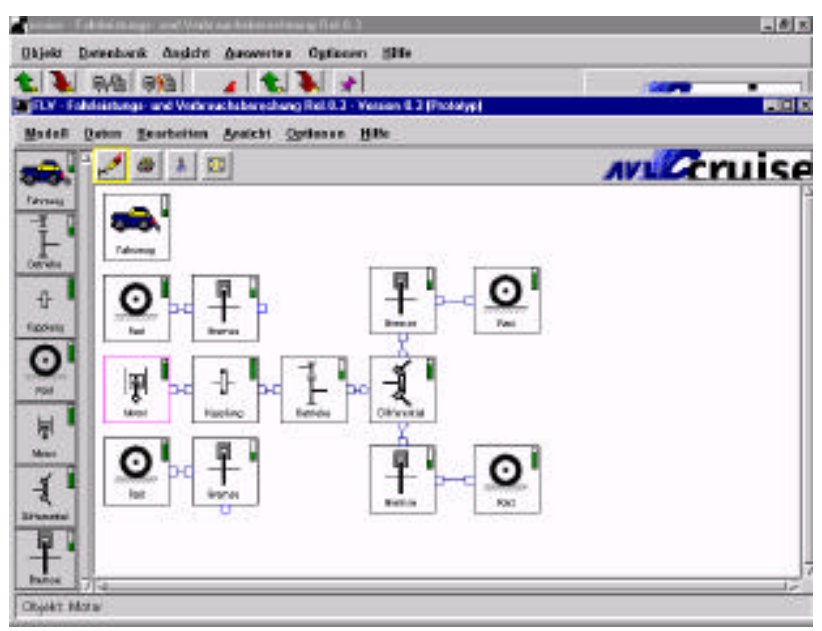

Figure 2: Offline Powertrain Simulation

\section{Hardware-in-the-Loop Simulation}

Once the conceptual decisions for a powertrain have been taken, the control systems of the engine and the transmission and the other vehicle systems resp. can already be combined - in parallel to the development of the components of the engine and the transmission or to the purchasing of these components.

In the so-called hardware-in-the-loop step, the realtime simulation is connected to the physically present control systems by means of the cable harness. If basic functions of the control unit, (e. g. gear shifting strategy block), have to be simulated within the offline simulation of the engine and the transmission, now the actual control units take over this. Furthermore, all the signals expected by the control systems have to be generated with the correct time related behavior.

In this stage, the following development applications are performed:

- Electronic integration test of the Engine Control Unit (ECU), the Transmission Control Unit (TCU), the cable harness, the sensors and actuators

- Communication test of the control units (CAN Bus)

- Functional developments (engine engagement at gear shifting, failure diagnosis)

- Automatic pre-calibrations and parameterization (On Board Diagnosis OBD)

As practice has shown, such trials and procedures are in the position to exclude failure sources and to shorten the overall testing time on the engine test bed or the powertrain test bed. An important additional aspect is the fact that all these applications and trials can be implemented in the "office laboratory" and do not take place on expensive test beds or in vehicles.

\section{Engine and Powertrain Test Bed}

As soon as the functionality of the control systems and their interaction have been ensured, they will be combined one with another on so-called powertrain test beds along with the components that have already been developed simultaneously, such as engine and transmission. These powertrain test beds represent multi-brake arrangements, on which the powertrain is mounted like a vehicle "skeleton" and only the body is missing.

However, this exactly is the advantage. For on such a skeleton, numerous measuring tasks can be fulfilled much better than in a complete vehicle. Measurements can be made independently from weather conditions, all around the clock and fully reproducible by the corresponding test bed control, critical driving states or entire driving cycles can be tested. The following work can be done on these highly specialized test beds:

- Mechanical / electronic integration tests

- Tests of driving performance

- Functional tests and verifications

- Parameterizations and calibrations [3],[4]

- Assessments of driveability [5] 
- Adjustments to the climate (with corresponding equipment)

- Covering emission test cycles, possibly with a CVS System

- Continuous runs for durability studies

- $\mathrm{NVH}$ (№ise, ibration, $\underline{\text { Harshness) studies }}$

The powertrain test bed is an ideal tool for assessing, developing and optimizing the interaction of all the components. In this development step again, the correction of malfunctions, the testing and refining of the operating strategies and the refilling of the data records in the control systems are top priorities.

In figure 3 a state-of-the-art powertrain test bed for front-wheel-drive and rear-wheel-drive con-figuration is shown, on which convential or even alternative drives like hybrid or fuell cell powertrains can be tested [6], [7].
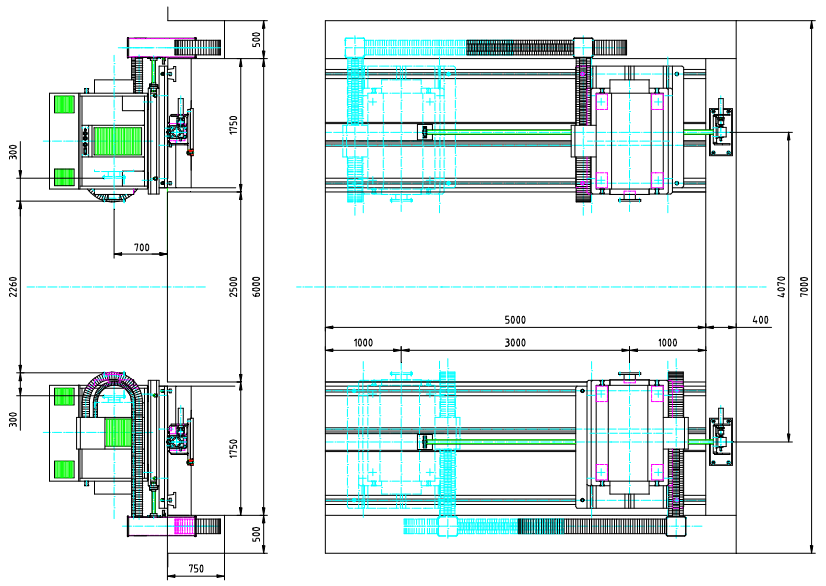

Figure 3: Powertrain Test Bed

For the testing of the combustion engine only with attached ECU the test bed platform AVL-ISAC 300 makes it possible to simulate transmissions not existing in reality by means of a highly dynamic asynchronous machine and corresponding models. By way of standard simulation models for automatic gear boxes and change speed gears are available. However, the extension of these models is made almost unlimited by an interface allowing real-time applications.

For example, two-mass oscillator models implemented in ISAC 300 can be extended by such modules as driver, foresighted driver, gear shifting strategies and simulation of continously variable transmission, which are calculated on an external simulation computer [8]. This makes it possible for the user to adapt these model components to his or her personal requirements and to configure the components correspondingly.

For example, it is possible to refine operating strategies specifically to the application. In this context, the numeric simulation program MATLAB/SIMULINK ${ }^{\circledast}$ is available to the user as development platform. Figure 4 shows an application in which the following components were modeled for a powertrain and in which a real-time processing within the test bed environment was implemented:

- Gear shifting strategy for automatic or manual gear box

- Braking strategies with retarder

- Driver and foresighted driver resp.

- Modeling of the auxiliary components like air condition

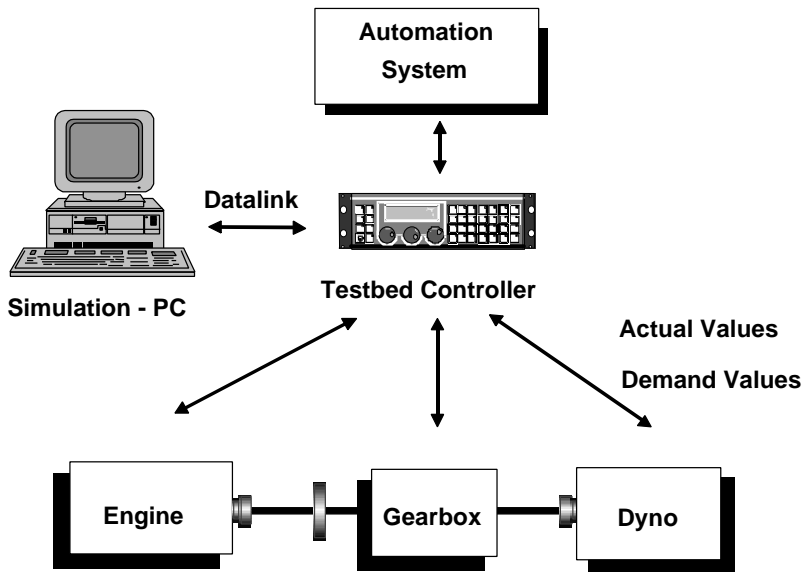

Figure 4: Highly-Dynamic Powertrain Test Bed with Open Simulation Models

For all test bed configurations a very realistic simulation of the driver and the test track is necessary in order to achive an operation of the powertrain on the test bed which is very close to the operation in the complete prototype vehicle. For this purpose specially designed editors to describe the geometric track properties and all driver information along this track is available. In figure 5 the graphical user interface of such an editor is shown. 


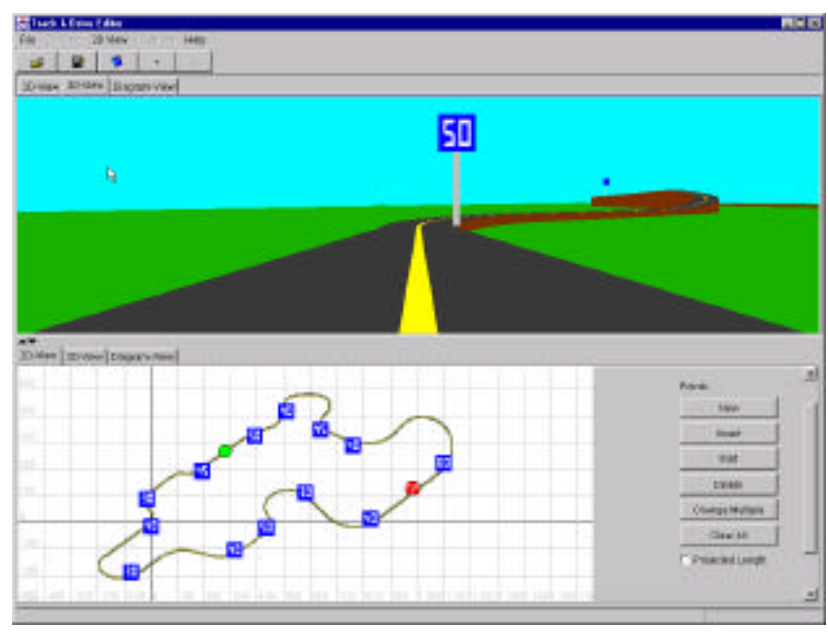

Figure 5: Graphical Track Editor

\section{In Vehicle Calibration}

As soon as the mechanical and electronic driving systems on the powertrain test bed have proved their quality, they will be installed in the vehicle. In this fourth and last stage, the required adjustments in the vehicle will be made:

- Road and chassis tests

- Verification of the driving performance

- Adjustment of driveability

- Height/summer/winter trials

- Finalizing the data records

- NVH optimizations

If all development steps in the previous stages have been kept and the simulation has been used as specified, according to experience the benefits take effect in the fourth phase. The vehicles can be developed with fewer prototypes, test rides and modification expenditure. Such a development process - once conceived and implemented consistently - will, due to the reduction in failure probability and frequency, fulfill the criteria in terms of saving cost and time and will also positively influence the quality of the final product and all the further developments.

\section{Summary}

A four-stage idealized development process for powertrain optimizations, which reflects the importance of accompanying simulation, has been presented. The use of simulation in all four essential development stages yields the following advantages:

- in the conceptual stage the certainty that the right decisions have been taken

- in the HIL (Hardware-in-the-Loop) stage the verification of the electronic systems, a task that is made increasingly difficult by the high complexity and the high degree of linking between the control systems

- in the development stage on the powertrain test bed the possibility to objectively verify the development stage and exclude malfunctions

- in the vehicle development stage a high saving potential provided by the fact that fewer prototypes and test rides are necessary.

If this development process is observed, a very good basis is laid for the further product development because the tools generated in this process can be put to further use without interruption.

Real-time simulation models of engines and transmissions, which can be adapted to the task in a flexible way, have been identified as very important tools for powertrain optimization using simulation.

The examples show that simulation can be used to optimize powertrain concepts and to refine the operating mode of existing powertrains. The integrated simple control unit test contributes to saving time and money in the development project.

As the development of simulation software is simultaneously done by several business areas within AVL, as competent development partners are integrated and as there is an in-house development for all types of engine and powertrain test beds, all customers are offered a unique and fully integrated development environment leading to a better quality of the products and helping to save time and money.

\section{References}

[1] Moser, F.; Kriegler, W.; Zrim, A.: Powertrain Optimization by Means of Simulation Tools, ATZ Automobiltechnische Zeitschrift, November 1999.

[2] Hasewend, W.; Herster, P.: Simulation of Vehicle Driving Performance, Fuel Consumption 
and Emissions. 31st ISATA, 2nd - 5th June, 1998, Düsseldorf, Germany.

[3] Gallacher, A.; Gschweitl, K.: Automated Dynamic Powertrain Calibration. Autotest '96', Barcelona 7/1996.

[4] Kampelmühler, F.; Paulitsch, R.; Gschweitl, K.: Automatic ECU Calibration - An Alternative to Conventional Methods. SAE Congress Detroit 3/1992.

[5] List, H.; Schoeggl, P.: Objective Evaluation of Vehicle Driveability. SAE 980204, Detroit, 1998.

[6] Zuber-Goos, F.: The BMW Research Test Bed for New Powertrain Concepts and its Requirements on the Simulation Technology, VDI Congress, Aachen Germany, September 1999.

[7] Schyr, C.: Advanced Simulation on the Testbed, JSAE Spring Convention, Yokohama Japan, May 1999.

[8] Juergens, G.; Gross, T.; Germann, S.; Abler, G.; Schyr, C.: CVT Simulation on the Dynamic Engine Test Bed, ATZ Automobiltechnische Zeitschrift, July 2000. 